ARTICLE

DOI: $10.1038 / s 41467-018-07482-6$

\title{
Efficient and stable sky-blue delayed fluorescence organic light-emitting diodes with $\mathrm{ClE}_{\mathrm{y}}$ below 0.4
}

Chin-Yiu Chan ${ }^{1}$, Masaki Tanaka ${ }^{1}$, Hajime Nakanotani ${ }^{1} \&$ Chihaya Adachi ${ }^{1}{ }^{12}$

Organic light-emitting diodes utilizing thermally activated delayed fluorescence is a potential solution for achieving stable blue devices. Sky-blue devices $\left(\mathrm{ClE}_{\mathrm{y}}<0.4\right)$ with high stability and high external quantum efficiency $(>15 \%)$ at $1000 \mathrm{~cd} \mathrm{~m}^{-2}$ based on either delayed fluorescence or phosphorescence are still limited and very hard to achieve simultaneously. Here, we report the design and synthesis of a new thermally activated delayed fluorescence emitter, $\mathbf{3} \mathbf{P h} \mathbf{}_{\mathbf{2}} \mathbf{C z} \mathbf{C} \mathbf{B} \mathbf{B N}$. A sky-blue device based on $\mathbf{3} \mathbf{P h}_{\mathbf{2}} \mathbf{C z} \mathbf{C} \mathbf{B} \mathbf{B N}$ exhibits a high external quantum efficiency of $16.6 \%$ at $1000 \mathrm{~cd} \mathrm{~m}^{-2}$. The device shows a sky-blue electroluminescence of $482 \mathrm{~nm}$ and achieves Commission Internationale de I' Eclairage coordinates of $(0.17,0.36)$. The sky-blue device exhibits a superb $L T_{90}$ of $38 \mathrm{~h}$. This is the first demonstration of high-efficiency and stable sky-blue devices $\left(\mathrm{CIE}_{\mathrm{y}}<0.4\right)$ based on delayed fluorescence, which represents an important advance in the field of blue organic lightemitting diode technology.

\footnotetext{
${ }^{1}$ Center for Organic Photonics and Electronics Research (OPERA), Kyushu University, 744 Motooka, Nishi, Fukuoka 819-0395, Japan. ${ }^{2}$ International Institute for Carbon Neutral Energy Research (WPI-I2CNER), Kyushu University, 744 Motooka, Nishi, Fukuoka 819-0395, Japan. Correspondence and requests for materials should be addressed to C.A. (email: adachi@cstf.kyushu-u.ac.jp)
} 
O rganic light-emitting diodes (OLEDs) are attractive technology for display and lighting due to their high flexibility, high efficiency and light-weight ${ }^{1-5}$. In order to commercialize OLEDs for various electronic applications, the color purity $^{6-11}$, efficiency ${ }^{12-15}$, driving voltage ${ }^{16-19}$ and device stability ${ }^{20-27}$ of OLEDs are some of the important parameters to be considered.

Blue and sky-blue phosphorescent OLEDs (PhOLEDs) with $\mathrm{CIE}_{\mathrm{y}}<0.4$ have been developing over 15 years $^{28}$, and the resultant PhOLEDs with high efficiencies and long operation times are still difficult to achieve simultaneously at high brightness ${ }^{17,18,20-22,28-}$ ${ }^{30}$. Regarding to the device stability, the $\mathrm{LT}_{90} \mathrm{~S}(90 \%$ of the initial luminance of $1000 \mathrm{~cd} \mathrm{~m}^{-2}$ ) of blue and sky-blue PhOLEDs are usually less than $10 \mathrm{~h}$, which are insufficient for commercialization $^{22,29,30}$. In 2014, Forrest and coworkers reported an improved stability of a single-unit blue phosphorescent OLEDs (dopant: Ir $\left.(\mathrm{dmp})_{3}\right)$ with the use of a graded-dopant-concentration technique in the emitting layer (EML) ${ }^{20}$. The graded device achieved a moderate external quantum efficiency (EQE) of $9.5 \%$ at $1000 \mathrm{~cd}$ $\mathrm{m}^{-2}$ with a driving voltage of $7.7 \mathrm{~V}$. Such devices exhibited a $\mathrm{LT}_{90}$ of $\sim 40 \mathrm{~h}$ at an initial luminance of $1000 \mathrm{~cd} \mathrm{~m}^{-2}$. Recently, in 2017, Forrest and coworkers further extended the device lifetime by introducing an extra molecule functioning as a hot excited-state manager in the EML of the graded devices ${ }^{21}$. The managed device achieved a similar EQE of $9.6 \%$ with a higher driving voltage of $9.0 \mathrm{~V}$ at $1000 \mathrm{~cd} \mathrm{~m}^{-2}$, and resulted in a lengthened $\mathrm{LT}_{90}$ of $\sim 140$ h. Nonetheless, the EQEs of both graded and managed devices were still less than $10 \%$ at $1000 \mathrm{~cd} \mathrm{~m}^{-2}$, rendering them less competitive with the commercial fluorescent blue emitters. Moreover, the driving voltages for both devices at $1000 \mathrm{~cd} \mathrm{~m}^{-2}$ were around $8 \mathrm{~V}$ or above, which were rather high for practical use.

Meanwhile, efficient OLEDs based on thermally activated delayed fluorescence (TADF) were first demonstrated by Adachi and coworkers in $2012^{31}$. TADF is believed to be a potential solution to achieve stable blue OLEDs with an internal quantum efficiency (IQE) of $100 \%$. Since then, there are numerous reports on efficient blue and sky-blue TADF OLEDs with $\mathrm{CIE}_{\mathrm{y}}<0.4^{32-34}$. However, reports on stable blue and sky-blue TADF OLEDs are still rare and limited ${ }^{23-25}$. Again, the $\mathrm{LT}_{90} \mathrm{~s}(90 \%$ of the initial luminance of $1000 \mathrm{~cd} \mathrm{~m}^{-2}$ ) of blue and sky-blue TADF OLEDs are always less than $10 \mathrm{~h}^{23,25}$. Recently, Adachi and coworkers reported a stable sky-blue TADF OLED by the use of a n-type host material (SF3-TRZ) ${ }^{24}$. The sky-blue TADF OLED was able to achieve a EQE of $7 \%$ with a driving voltage of $5.7 \mathrm{~V}$ at $1000 \mathrm{~cd}$ $\mathrm{m}^{-2}$. The corresponding device exhibited a comparable $\mathrm{LT}_{90}$ of $\sim 20 \mathrm{~h}$ (at an initial luminance of $1000 \mathrm{~cd} \mathrm{~m}^{-2}$ ) to those reported sky-blue PhOLEDs ${ }^{20,21}$. However, same as PhOLEDs ${ }^{20,21}$, the EQE of the stable sky-blue TADF OLED was still below $10 \%$. As a result, there is still a room for designing new TADF emitters to concurrently achieve blue or sky-blue OLEDs with a high EQE and a high stability at high brightness.

Here we show that a sky-blue device based on a new fluorescence emitter $\left(\mathbf{3 P h}_{\mathbf{2}} \mathbf{C z C z B N}\right)$ exhibits a high external quantum efficiency of $16.6 \%$ at $1000 \mathrm{~cd} \mathrm{~m}^{-2}$. The device shows a sky-blue electroluminescence of $482 \mathrm{~nm}$ and achieves Commission Internationale de l' Eclairage coordinates of $(0.17,0.36)$. The sky-blue device exhibits a superb $\mathrm{LT}_{90}$ of $38 \mathrm{~h}$. This is the first demonstration of high-efficiency $(>15 \%)$ and stable sky-blue devices $\left(\mathrm{CIE}_{\mathrm{y}}<0.4\right)$ based on delayed fluorescence at $1000 \mathrm{~cd} \mathrm{~m}^{-2}$.

\section{Results}

Molecular design and synthesis. The long-lived triplet excitons are believed to be lethal to the device stability for both phosphorescent and TADF OLEDs, because hot triplet excitons are generated by triplet-triplet annihilation or triplet-polaron annihilation processes $35-37$. Therefore, decreasing the delayed lifetimes of the blue TADF emitters is useful to enhance the device stability. Benzonitrile is one of the promising building blocks for constructing stable blue TADF emitters ${ }^{23}$. However, blue benzonitrile-based TADF emitters usually possess long delayed lifetimes, thus resulting in severe rolloffs and short device lifetimes in OLEDs ${ }^{23}$. Recently, it is proven that the introduction of two different donor units in the TADF emitters is effective to lower the energy of the high-lying localized triplet excited state $\left({ }^{3} \mathrm{LE}\right)$, in which a good mixing of the ${ }^{3} \mathrm{LE}$ state with the lowest charge transfer triplet excited state $\left({ }^{3} \mathrm{CT}\right)$ can facilitate a faster reverse intersystem crossing rate $\left(k_{\mathrm{RISC}}\right)^{38}$. Moreover, such faster $k_{\mathrm{RISC}}$ results in a shorter delayed lifetime of the TADF emitters, which eventually improves the device stability since triplet exciton intensity in devices will be reduced. Herein, we synthesized a new blue benzonitrile-based TADF emitter, $\mathbf{3} \mathbf{P h}_{\mathbf{2}} \mathbf{C z C z B N}$, in two steps (Fig. 1). 4CzBN was also synthesized in one step as a reference ${ }^{23}$. Both compounds were well characterized by ${ }^{1} \mathrm{H}$ and ${ }^{13} \mathrm{C} \mathrm{NMR}$, mass spectrometry analysis and elemental analysis (Supplementary Figures 1-7). Their purities were also confirmed by high-performance liquid chromatography (Supplementary Figures 8 and 9). It is expected that the hetero-donor-based TADF emitter $\left(\mathbf{3} \mathbf{P h}_{\mathbf{2}} \mathbf{C z C z B N}\right)$ should show a shorter delayed lifetime and a longer device lifetime than that of the homo-donorbased reference compound (4CzBN). Quantum-chemical calculations based on $\mathbf{3} \mathbf{P h}_{\mathbf{2}} \mathbf{C z C z B N}$ and $\mathbf{4 C z B N}$ were first performed by time-dependent density functional theory (TD-DFT) at B3LYP/6-31 G(d) level. The calculated highest occupied molecular orbital (HOMO), lowest unoccupied molecular orbital (LUMO), singlet $\left(\mathrm{S}_{1}\right)$ and triplet $\left(\mathrm{T}_{1}\right)$ energy levels, oscillator strength $(f)$ and $\Delta E_{\mathrm{ST}} s$ were depicted in Fig. 1. From the TD-DFT calculation, $3 \mathbf{P h}_{2} \mathrm{CzCzBN}$ and $4 \mathrm{CzBN}$ showed similar $\Delta E_{\mathrm{ST}} \mathrm{s}$ of 0.13 and $0.14 \mathrm{eV}$ and high oscillator strengths $\left(f_{\mathrm{s}}\right)$ of 0.0619 and 0.0818 , respectively. The calculated HOMOs of both compounds were mainly localized on the carbazole donors with some extensions on the benzonitrile acceptor, whereas the LUMOs are fully localized on the benzonitrile acceptor.

Photophysical, electrochemical and thermal properties. The photophysical properties of $\mathbf{3} \mathbf{P h}_{\mathbf{2}} \mathbf{C z C z B N}$ and $\mathbf{4 C z B N}$ were first examined in toluene solution at the concentration of $10^{-5} \mathrm{M}$. Both compounds showed absorption bands from 290 to $450 \mathrm{~nm}$, in which the absorption bands at around $400-450 \mathrm{~nm}$ were assigned as the intramolecular charge transfer (ICT) bands (Fig. 2). As expected, by the introduction of 3,6-diphenylcarbazole, $3 \mathbf{P h}_{2} \mathbf{C z C z B N}$ showed a redshifted absorption than that of $\mathbf{4 C z B N}$. The energy gaps of $\mathbf{3} \mathbf{P h}_{2} \mathbf{C z C z B N}$ and $\mathbf{4 C z B N}$ were calculated from their onset values of $10 \%$ of the absorption maxima and were found to be 2.79 and $2.89 \mathrm{eV}$, respectively. Upon photo-excitation at the ICT band, $3 \mathbf{P h}_{\mathbf{2}} \mathbf{C z C z B N}$ and 4CzBN both showed structureless emissions in the blue region with the peak maxima of 464 and $443 \mathrm{~nm}$, respectively (Fig. 2 and Table 1). The redshifted absorption and emission spectrum of $3 \mathbf{P h}_{2} \mathbf{C z C z B N}$ were consistent to the TD-DFT calculation. The $\mathrm{T}_{1} \mathrm{~s}$ of $3 \mathbf{P h}_{2} \mathbf{C z C z B N}$ and $\mathbf{4 C z B N}$ were found to be 2.60 and 2.66 $\mathrm{eV}$, which were estimated from the phosphorescence spectra in toluene solutions at $77 \mathrm{~K}$ (Fig. 2 and Table 1 ). The $\Delta E_{\mathrm{ST}} \mathrm{s}$ of $\mathbf{3 P h}_{\mathbf{2}} \mathbf{C z C z B N}$ and $\mathbf{4 C z B N}$ were calculated to be 0.19 and $0.23 \mathrm{eV}$, respectively. The photoluminescence quantum yields (PLQYs) of $3 \mathrm{Ph}_{2} \mathrm{CzCzBN}$ and $4 \mathrm{CzBN}$ were found to be 16 and $9 \%$, respectively, before degassing. After purging $\mathrm{N}_{2}$ into the solutions, the corresponding PLQYs of $\mathbf{3} \mathbf{P h}_{\mathbf{2}} \mathbf{C z C z B N}$ and $\mathbf{4 C z B N}$ were greatly enhanced and found to be 95 and $63 \%$ (Table 1). Doping $15 \mathrm{wt} \%$ of $3 \mathbf{P h}_{2} \mathbf{C z C z B N}$ and $\mathbf{4 C z B N}$ in 3,3'-di(9H-carbazol-9-yl)-1,1'- 
a

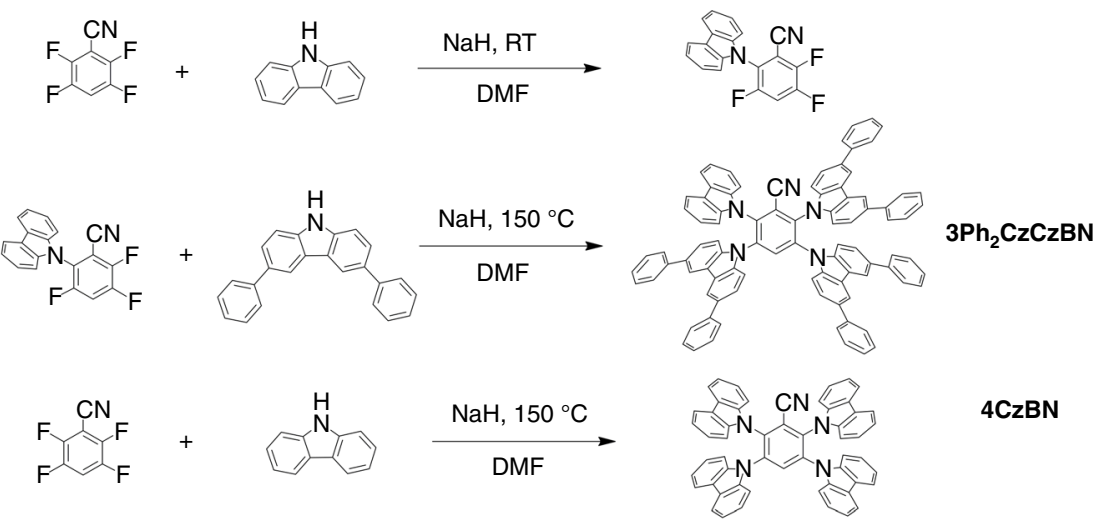

b
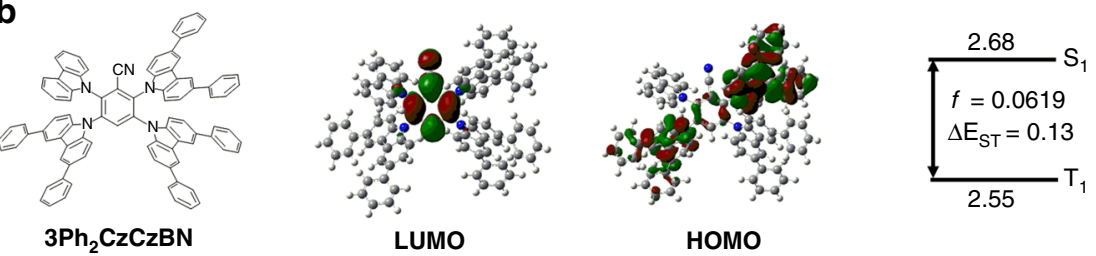

LUMO

HOMO
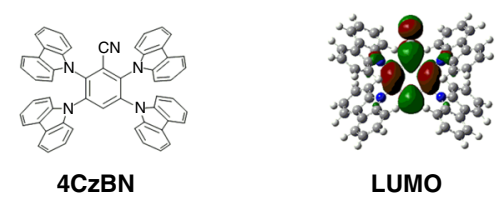

LUMO
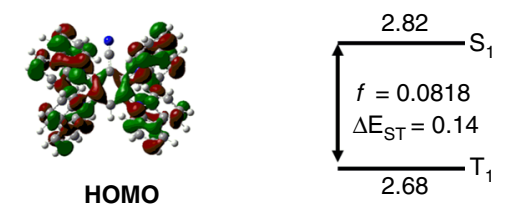

Fig. 1 Synthetic scheme and quantum chemical calculations of the blue emitters. a Synthetic scheme of $\mathbf{3} \mathbf{P h} \mathbf{2} \mathbf{C z} \mathbf{C z} \mathbf{B N}$ and $\mathbf{4} \mathbf{C z} \mathbf{B N}$. $\mathbf{b}$ Quantum chemical calculations of $\mathbf{3} \mathbf{P h}_{\mathbf{2}} \mathbf{C z} \mathbf{C z B N}$ and $\mathbf{4 C z B N}$

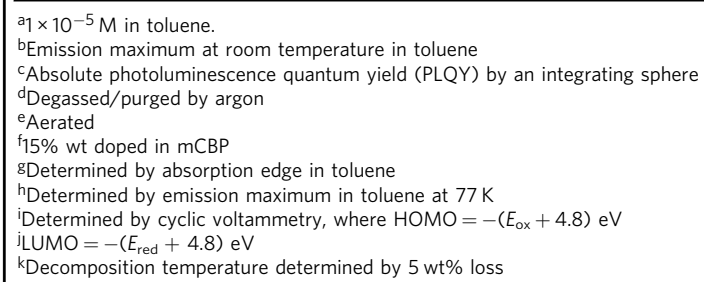

${ }^{k}$ Decomposition temperature determined by $5 \mathrm{wt} \%$ loss

biphenyl (mCBP) resulted in slightly lower PLQYs of 91 and 51\% (Supplementary Table 1).

The transient decay profiles of $\mathbf{3} \mathbf{P h}_{\mathbf{2}} \mathbf{C z C z B N}$ and $\mathbf{4} \mathbf{C z B N}$ were first studied in degassed toluene solution. The prompt lifetimes of $3 \mathrm{Ph}_{\mathbf{2}} \mathrm{CzCzBN}$ and $4 \mathrm{CzBN}$ were found to be 3.7 and $1.6 \mathrm{~ns}$, whereas the delayed lifetimes were found to be 10 and $50 \mu$ s, respectively (Supplementary Figure 10). Notably, the delayed lifetime of $\mathbf{3} \mathbf{P h}_{\mathbf{2}} \mathbf{C z C z B N}$ was greatly shortened comparing to that of $4 \mathrm{CzBN}$, which may be ascribed to its smaller $\Delta E_{\mathrm{ST}}$. The TADF properties of $\mathbf{3} \mathbf{P h}_{\mathbf{2}} \mathbf{C z C z B N}$ and $\mathbf{4} \mathbf{C z B N}$ in the thin films were also studied by the streak camera. Simply doping $15 \mathrm{wt} \%$ of $\mathbf{3 P h} \mathbf{P}_{\mathbf{2}} \mathbf{C z C z B N}$ or $\mathbf{4 C z B N}$ in $\mathrm{mCBP}$, the delayed lifetimes at $300 \mathrm{~K}$ were found to be 5.2 and $13.7 \mu$ s (Supplementary Figure 11), which were consistent to that in the solution state. The TADF behaviors were confirmed by performing temperature-dependent transient decay study of the $15 \mathrm{wt} \%$ doped films from 50 to $300 \mathrm{~K}$ (Fig. 2 and Supplementary Figures 11, 12 and 14). The detailed rate constants were also calculated and tabulated in Supplementary Table 1 . The activation energies $\left(\Delta E_{a}^{\mathrm{TADF}}\right)$ for the delayed fluorescence were also calculated from the Arrhenius plots of $k_{\mathrm{RISC}}$ vs. $1 / T$ based on the relationship $k_{\mathrm{RISC}}=\exp ($ $\left.-\Delta E_{a}^{\mathrm{TADF}} / k_{\mathrm{B}} T\right)$, where $k_{\mathrm{RISC}}$ is the rate constant of reverse intersystem crossing, $k_{\mathrm{B}}$ is the Boltzmann's constant and $\mathrm{T}$ is the temperature. The $\Delta E_{a}^{\mathrm{TADF}} \mathrm{s}$ of $\mathbf{3} \mathbf{P h}_{\mathbf{2}} \mathbf{C z C z B N}$ and $\mathbf{4 C z B N}$ were found to be 42 and $100 \mathrm{meV}$, respectively (Supplementary Table 2 and 3, Supplementary Figures 13 and 15). Also, the $k_{\text {RISC }}$ of $3 \mathbf{P h}_{2} \mathbf{C z C z B N}$ and $\mathbf{4 C z B N}$ were found to be $4.44 \times 10^{5}$ and $2.15 \times 10^{5} \mathrm{~s}^{-1}$, respectively (Supplementary Table 1 ). The smaller $\Delta E_{a}^{\mathrm{TADF}}$ of $\mathbf{3} \mathbf{P h}_{\mathbf{2}} \mathbf{C z C z B N}$ may account for the faster $k_{\mathrm{RISC}}$.

The electrochemical properties of $3 \mathbf{P h}_{\mathbf{2}} \mathbf{C z C z B N}$ and $\mathbf{4 C z B N}$ were determined by cyclic voltammetry in $\mathrm{N}, \mathrm{N}$-dimethylformamide solutions (Supplementary Figures 16-19). The HOMOs of $3 \mathbf{P h}_{2} \mathbf{C z C z B N}$ and $\mathbf{4 C z B N}$ were determined to be -5.69 and $-5.79 \mathrm{eV}$, respectively, whereas their corresponding LUMOs were found to be -2.78 and $-2.75 \mathrm{eV}$. The HOMOs of $\mathbf{3} \mathbf{P h}_{\mathbf{2}} \mathbf{C z C z B N}$ and $4 \mathrm{CzBN}$ were also estimated by measuring their neat films with the photoelectron spectrometer. Their HOMOs were found to be -5.91 and $-5.99 \mathrm{eV}$, respectively (Supplementary Figures 20 
a

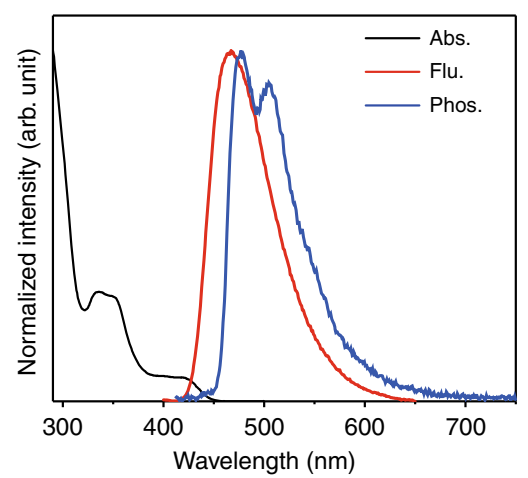

C

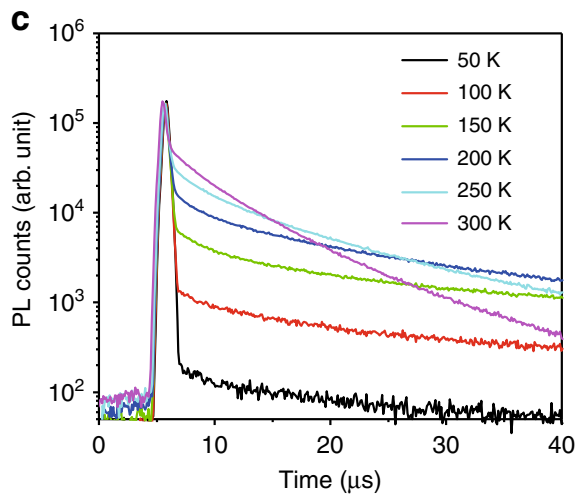

e

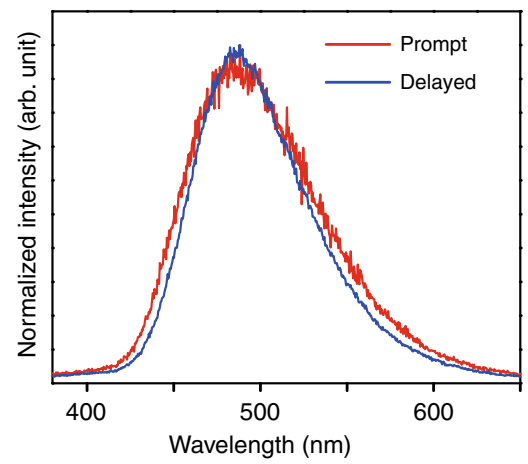

b

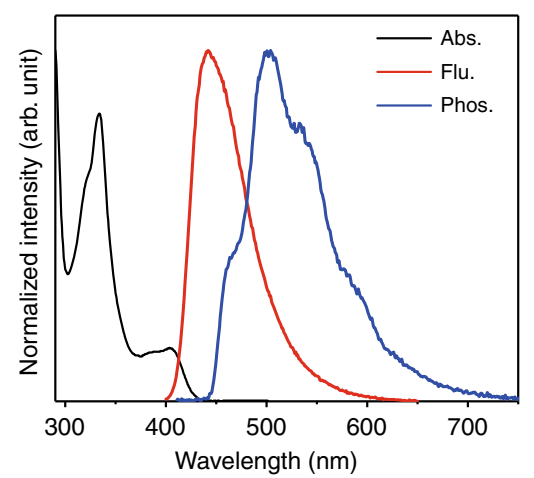

d

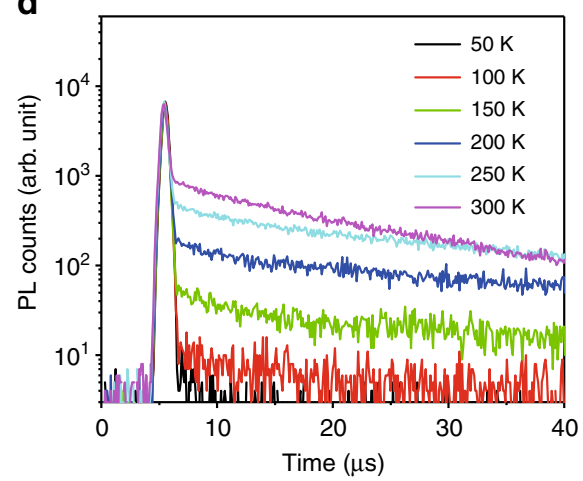

f

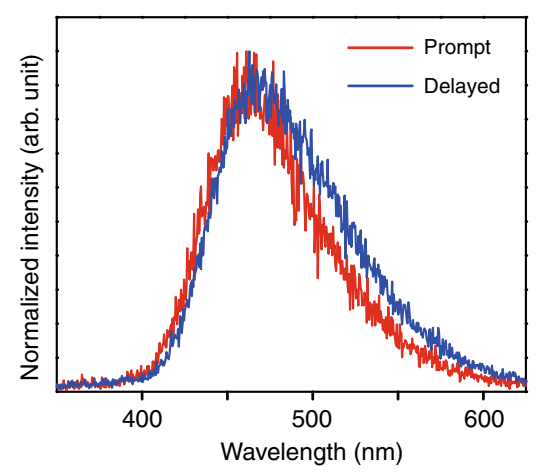

Fig. 2 Photophysical properties of $\mathbf{3} \mathbf{P h}_{\mathbf{2}} \mathbf{C z} \mathbf{C z} \mathbf{B N}$ and $\mathbf{4} \mathbf{C z} \mathbf{B N}$. a Absorption, fluorescence and phosphorescence spectra of $\mathbf{3} \mathbf{P h}{ }_{\mathbf{2}} \mathbf{C z} \mathbf{C z} \mathbf{B N}$ in toluene. $\mathbf{b}$ Absorption, fluorescence and phosphorescence spectra of $\mathbf{4 C z B N}$ in toluene. $\mathbf{c}$ Temperature-dependence transient decay profiles of a thin film of 15 wt $\%$ of $\mathbf{3 P h}_{\mathbf{2}} \mathbf{C z} \mathbf{C z B N}$ doped in mCBP. $\mathbf{d}$ Temperature-dependence transient decay profiles of a thin film of $15 \mathrm{wt} \%$ of $\mathbf{4 C z} \mathbf{B N}$ doped in mCBP. $\mathbf{e}$ Prompt and delayed spectra of a thin film of $15 \mathrm{wt} \%$ of $\mathbf{3} \mathbf{P h}_{\mathbf{2}} \mathbf{C z} \mathbf{C z} \mathbf{B N}$ doped in mCBP. $\mathbf{f}$ Prompt and delayed spectra of a thin film of 15 wt $\%$ of $\mathbf{4 C z} \mathbf{B N}$ doped in mCBP

and 21). Furthermore, the thermogravimetry - differential thermal analysis (TG-DTA) and differential scanning calorimetry (DSC) analysis were also performed to examine the thermal properties of $\mathbf{3} \mathbf{P h}_{\mathbf{2}} \mathbf{C z C z B N}$ and $\mathbf{4 C z B N}$. From TG-DTA measurement, $\mathbf{3} \mathbf{P h}_{\mathbf{2}} \mathbf{C z C z B N}$ and $\mathbf{4 C z B N}$ showed excellent thermal stability with $\mathrm{T}_{\mathrm{d}} \mathrm{s}$ ( $5 \%$ weight loss) of $>500$ and $455^{\circ} \mathrm{C}$, respectively (Supplementary Figures 22 and 23). The melting points of the sublimed samples were found to be 433 and $448^{\circ} \mathrm{C}$, respectively (Supplementary Figures 22 and 23). From DSC measurement, $\mathbf{3} \mathbf{P h}_{\mathbf{2}} \mathbf{C z C z B N}$ showed glass transition temperature $\left(\mathrm{T}_{\mathrm{g}}\right)$ at $240^{\circ} \mathrm{C}$ (Supplementary Figure 24). The DSC measurement of $4 \mathrm{CzBN}$ was not performed, since its melting point is close to its $\mathrm{T}_{\mathrm{d}}$. All the photophysical, electrochemical and thermal properties were tabulated in Table 1 and Supplementary Table 1.

Device characterization and performance. To evaluate the electroluminescence (EL) properties of $3 \mathbf{P h}_{2} \mathbf{C z C z B N}$ and $4 \mathrm{CzBN}$, multilayered OLEDs (device $\mathbf{A}$ and $\mathbf{B}$ ) were fabricated using the following structure: Indium-tin oxide (ITO)-coated glass $(100 \mathrm{~nm}) /$ HATCN $(10 \mathrm{~nm}) /$ Tris-PCz $(30 \mathrm{~nm}) / \mathrm{mCBP}(5 \mathrm{~nm}) / 15 \mathrm{wt} \%$ of $3 \mathbf{P h}_{2} \mathbf{C z C z B N}$ (device $\mathbf{A}$ ) or $15 \mathrm{wt} \%$ of $\mathbf{4 C z B N}$ (device $\mathbf{B}$ ): $\mathrm{mCBP}$ $(30 \mathrm{~nm}) / \mathrm{T} 2 \mathrm{~T}(10 \mathrm{~nm}) / \mathrm{BPy}-\mathrm{TP} 2(40 \mathrm{~nm}) / \mathrm{LiF}(0.8 \mathrm{~nm}) / \mathrm{Al}(100$ $\mathrm{nm})$, in which hexaazatriphenylenehexacarbonitrile (HATCN) was the hole-injection layer, 9,9',9"-triphenyl-9H,9' $H, 9^{\prime \prime} \mathrm{H}-2,3^{\prime}: 6^{\prime}, 2^{\prime \prime}$ tercarbazole (Tris-PCz) was the hole-transporting layer, 3,3'-di $(9 H$ carbazol-9-yl)-1,1'-biphenyl (mCBP) was theelectron-blocking layer, $15 \mathrm{wt} \%$ of $\mathbf{3} \mathbf{P h}_{\mathbf{2}} \mathbf{C z C z B N}$ (device $\mathbf{A}$ ) or $15 \mathrm{wt} \%$ of $\mathbf{4 C z B N}$ (device $\mathbf{B}$ ) doped in $\mathrm{mCBP}$ was the emitting layer (EML), 2,4,6-tris(biphenyl3-yl)-1,3,5-triazine (T2T) was the hole-blocking layer, 2,7-di(2,2'bipyridin-5-yl)triphenylene (BPy-TP2) was the electrontransporting layer and $\mathrm{LiF}$ and $\mathrm{Al}$ were the electron injection layer and cathode, respectively. The materials used and the device structure for devices A and B were depicted in Supplementary Figures 25 and 26.

The detailed OLEDs performance for devices $\mathbf{A}$ and $\mathbf{B}$ were shown in Supplementary Figures 27-34 and Table 2. Both devices A and B showed sky-blue emission with maximum peaks at 482 
and $477 \mathrm{~nm}$, respectively. The CIE coordinates of device $\mathbf{A}$ and $\mathbf{B}$ were found to be $(0.17,0.37)$ and $(0.19,0.32)$, respectively. Device A showed a maximum EQE of $15.9 \%$ and dropped to $15.0 \%$ at $1000 \mathrm{~cd} \mathrm{~m}^{-2}$, whereas device $\mathbf{B}$ showed a severe rolloff with a maximum EQE of $9.7 \%$ and a much lower EQE of only $5.8 \%$ at $1000 \mathrm{~cd} \mathrm{~m}^{-2}$. The driving voltages of devices $\mathbf{A}$ and $\mathbf{B}$ were found to be 6.3 and $7.6 \mathrm{~V}$ at $1000 \mathrm{~cd} \mathrm{~m}^{-2}$, respectively. Devices $\mathbf{A}$ and $\mathbf{B}$ were driven at a constant DC current at room temperature for testing the device stability. The $\mathrm{LT}_{90} \mathrm{~s}$ of devices $\mathbf{A}$ and $\mathbf{B}$ were found to be 4 and $0.1 \mathrm{~h}$, respectively. Hole-only and electron-only devices (HOD and EOD) based on the structures of devices $\mathbf{A}$ and B were also fabricated to understand more about the carrier transport properties (Supplementary Figure 35). It was found that both devices showed similar carrier transport properties. For $3 \mathrm{Ph}_{2} \mathbf{C z C z B N}$, the current density ratios of HOD to EOD at 6 and $7 \mathrm{~V}$ were 20 and 57, respectively. Meanwhile, for $4 \mathbf{C z B N}$, the current density ratios of HOD to EOD at 6 and $7 \mathrm{~V}$ were 14 and 47 , respectively. It seemed that the carrier transport properties in device $\mathbf{A}$ and $\mathbf{B}$ were not the major determining factor for the stability enhancement. In fact, we believed the enhanced stability of device $\mathbf{A}$ was originated from the shortening of the delayed lifetime of $\mathbf{3} \mathbf{P h}_{\mathbf{2}} \mathbf{C z C z B N}(5.2 \mu \mathrm{s})$ than that of $\mathbf{4} \mathbf{C z B N}(13.7 \mu \mathrm{s})$. The shortening of the delayed lifetime of $\mathbf{3} \mathbf{P h}_{\mathbf{2}} \mathbf{C z C z B N}$ lowered the triplet exciton intensity in EML, in which the rolloff in device A was significantly reduced. Moreover, the diminished triplet exciton intensity also avoided exciton-exciton annihilation and exciton-polaron annihilation, thus enhancing the device stability.

To further improve the device stability, devices $\mathbf{C}$ and $\mathbf{D}$ were fabricated with the following device structure: Indium-tin oxide (ITO)-coated glass $(100 \mathrm{~nm}) / \mathrm{HATCN}(10 \mathrm{~nm}) / \mathrm{Tris}-\mathrm{PCz}(30$ $\mathrm{nm}) / \mathrm{mCBP}(5 \mathrm{~nm}) / 15 \mathrm{wt} \%$ of $\mathbf{3} \mathbf{P h} \mathbf{h}_{2} \mathbf{C z C z B N}$ (device C) or $20 \mathrm{wt}$ $\%$ of $3 \mathbf{P h}_{2} \mathbf{C z C z B N}$ (device D): $\mathrm{mCBP}(30 \mathrm{~nm}) / \mathrm{SF} 3-\mathrm{TRZ}(10$ $\mathrm{nm}) / 30 \mathrm{wt} \%$ of Liq: SF3-TRZ $(50 \mathrm{~nm}) / \mathrm{Liq}(2 \mathrm{~nm}) / \mathrm{Al}(100 \mathrm{~nm})$, in which HATCN was the hole-injection layer, Tris-PCz was the hole-transporting layer, mCBP was the electron-blocking layer, $15 \mathrm{wt} \%$ of $\mathbf{3} \mathbf{P h}_{\mathbf{2}} \mathbf{C z C z B N}$ (device $\mathbf{C}$ ) or $20 \mathrm{wt} \%$ of $\mathbf{3} \mathbf{P h}_{\mathbf{2}} \mathbf{C z C z B N}$ (device D) doped in mCBP was the emitting layer (EML), 2-(9,9'spirobi[fluoren]-6-yl)-4,6-diphenyl-1,3,5-triazine (SF3-TRZ) was the hole-blocking layer, $30 \mathrm{wt} \%$ of 8 -quinolinolato lithium (Liq): SF3-TRZ was the electron-transporting layer and Liq and $\mathrm{Al}$ were the electron injection layer and cathode.

Comparing to device $\mathbf{A}$, device $\mathbf{C}$ showed the identical EL peak maximum of $482 \mathrm{~nm}$ with the CIE coordinates of $(0.18,0.37)$. Device $\mathbf{C}$ also showed a same maximum EQE of $15.9 \%$. The turnon voltage of device $\mathbf{C}$ was $3.7 \mathrm{~V}$, which was lower than that of device $\mathbf{A}(4.0 \mathrm{~V})$. Furthermore, the driving voltage of device $\mathbf{C}$ was found to be $6.4 \mathrm{~V}$ at $1000 \mathrm{~cd} \mathrm{~m}^{-2}$. Although device $\mathbf{C}$ showed a slightly lower EQE of $14.3 \%$ at $1000 \mathrm{~cd} \mathrm{~m}^{-2}$, the $\mathrm{LT}_{90}$ of device $\mathrm{C}$ was significantly improved by four times to $16 \mathrm{~h}$. Further optimizing the device performance, the doping concentration of $\mathbf{3 P h}_{\mathbf{2}} \mathbf{C z C z B N}$ was increased from $15 \mathrm{wt} \%$ (device C) to $20 \mathrm{wt} \%$ (device $\mathbf{D}$ ). In device $\mathbf{D}$, the EL peak maximum was mildly redshifted to $486 \mathrm{~nm}$ with CIE coordinates of $(0.18,0.39)$. Device D showed a maximum EQE of $17.9 \%$. Interestingly, device D showed an insignificant rolloff and achieved a EQE of $17.2 \%$ at $1000 \mathrm{~cd} \mathrm{~m}^{-2}$. Meanwhile, a driving voltage of $5.8 \mathrm{~V}$ was obtained. Most importantly, the $\mathrm{LT}_{90}$ of device $\mathbf{D}$ was lengthened to $32 \mathrm{~h}$, which is a 320 -times increase comparing to that of the $4 \mathbf{C z B N}$ based device. The detailed device characteristics of device $\mathbf{C}$ and $\mathbf{D}$ were depicted in Fig. 3 and tabulated in Table 2. Additionally, color tuning based on device $\mathbf{D}$ for achieving bluer emission is possible by optimizing the thicknesses of each layer or by the use of micro-cavity effect and/or filters.

An optical simulation based on device $\mathbf{D}$ has been performed to study the effect of the thickness of the ETL $(10$ to $60 \mathrm{~nm})$ on the EL emission color (Supplementary Figure 36). It was found that the EL emission would be blue-shifted with the thinner ETL layer. However, too thin ETL may result in imbalanced carrier mobilities of the device. Therefore, an attempt on reducing the thickness of the ETL from $50 \mathrm{~nm}$ (device D) to $20 \mathrm{~nm}$ (device E) was performed. Device $\mathbf{E}$ resulted in comparable EQEs of $17.8 \%$ and $16.6 \%$ at the maximum value and $1000 \mathrm{~cd} \mathrm{~m}^{-2}$, respectively. Also, device $\mathbf{E}$ showed a slightly lengthened stability with a $\mathrm{LT}_{90}$ of $38 \mathrm{~h}$. Most importantly, device $\mathbf{E}$ achieved better CIE coordinates of $(0.17,0.36)$ at $1000 \mathrm{~cd} \mathrm{~m}^{-2}$, which is bluer than that of $(0.18,0.39)$ in device $\mathbf{D}$ (Table 2 and Supplementary Figures 37-40).

A summary of the best reported blue and sky-blue OLEDs based on delayed fluorescence and phosphorescence was listed in Supplementary Table 4 . To our best knowledge, the result present here is the first report of a sky-blue OLED $\left(\mathrm{CIE}_{\mathrm{y}}<0.4\right)$ with a high external quantum efficiency (EQE 16.6\%) and a long stability $\left(\mathrm{LT}_{90} \sim 38 \mathrm{~h}\right)$ at high brightness $\left(1000 \mathrm{~cd} \mathrm{~m}^{-2}\right)$, which represents a big milestone to the field of the blue OLED technology.

\section{Discussion}

A new TADF emitter, $\mathbf{3} \mathbf{P h}_{\mathbf{2}} \mathbf{C z C z B N}$, has been synthesized and characterized. It showed a higher PLQY and a shorter delayed lifetime than that of the reference compound, $4 \mathbf{C z B N}$. Devices based on $3 \mathbf{P h}_{2} \mathbf{C z C z B N}$ showed a sky-blue EL of $482 \mathrm{~nm}$ and CIE coordinates of $(0.17,0.36)$. Such device achieved a high EQE of $16.6 \%$ at $1000 \mathrm{~cd} \mathrm{~m}^{-2}$. Most importantly, $3 \mathbf{P h}_{\mathbf{2}} \mathbf{C z C z B N}$-based TADF OLEDs showed superior device stability. With an initial luminance of $1000 \mathrm{~cd} \mathrm{~m}^{-2}$, an exceptional $\mathrm{LT}_{90}$ of $38 \mathrm{~h}$ was achieved. In conclusion, a stable sky-blue OLED $\left(\mathrm{CIE}_{\mathrm{y}}<0.4\right)$ and a high external quantum efficiency $(>15 \%)$ at high brightness $\left(1000 \mathrm{~cd} \mathrm{~m}^{-2}\right)$ were achieved simultaneously with the use of a newly synthesized TADF emitter, $\mathbf{3 P h}_{\mathbf{2}} \mathbf{C z C z B N}$. This outstanding result definitely suggests the possibility for achieving efficient and stable blue OLEDs by TADF technology.

\section{Methods \\ General. All reagents were used as received from commercial sources and were used without further purification. Chromatographic separations were carried out using silica gel $(200-300 \mathrm{~nm})$. The two materials investigated in this paper were synthesized by following the procedures described below. All compounds were purified twice by temperature gradient vacuum sublimation. ${ }^{1} \mathrm{H},{ }^{13} \mathrm{C}$ and ${ }^{19} \mathrm{~F}$ nuclear magnetic resonance (NMR) spectra were obtained in $\mathrm{CDCl}_{3}$ or acetone- $\mathrm{d}_{6}$ with a Bruker Biospin Avance-III 500 NMR spectrometer at ambient temperature. Chemical shifts $(\delta)$ are given in parts per million (ppm) relative to tetra- methylsilane (TMS; $\delta=0$ ) as the internal reference. Mass spectra were measured in positive-ion atmospheric-pressure chemical ionization (APCI) mode on a Waters 3100 mass detector. Elemental analyses $(\mathrm{C}, \mathrm{H}$ and $\mathrm{N}$ ) were carried out with a Yanaco MT-5 elemental analyzer.}

Synthesis of $\mathbf{3} \mathbf{P h}_{\mathbf{2}} \mathbf{C z} \mathbf{C z B N}$. Under nitrogen atmosphere, 3,6-diphenyl-9H-carbazole $(957 \mathrm{mg}, 3 \mathrm{mmol})$ was dissolved in dry $N, N$-dimethylformamide $(30 \mathrm{~mL})$ in a two-neck round-bottom flask equipped with a condenser. The reaction mixture was cooled to $0{ }^{\circ} \mathrm{C}$, then $\mathrm{NaH}(120 \mathrm{mg}, 3 \mathrm{mmol})$ was added. The reaction mixture was slowly warmed to room temperature and stirred for half an hour. After that, 2 (9H-Carbazol-9-yl)-3,5,6-trifluorobenzonitrile (322 mg, $1 \mathrm{mmol}$ ) was added and the reaction was heated to $150^{\circ} \mathrm{C}$ for $16 \mathrm{~h}$. The reaction was quenched with water and the precipitate was filtered off. The crude product was purified by column chromatography. Yield: $976 \mathrm{mg}(80 \%) .{ }^{1} \mathrm{H}$ NMR $\left(500 \mathrm{MHz}\right.$, Acetone- $\mathrm{d}_{6}, 298 \mathrm{~K}$, relative to $\left.\mathrm{Me}_{4} \mathrm{Si}\right): \delta=9.07(\mathrm{~s}, 1 \mathrm{H}), 8.36(\mathrm{~s}, 2 \mathrm{H}), 8.29(\mathrm{~d}, 4 \mathrm{H}, 10.0 \mathrm{~Hz}), 7.95-8.00$ $(\mathrm{m}, 10 \mathrm{H}), 7.55-7.70(\mathrm{~m}, 18 \mathrm{H}), 7.30-7.45(\mathrm{~m}, 20 \mathrm{H}), 7.18(\mathrm{t}, 2 \mathrm{H}, 7.0 \mathrm{~Hz}) .{ }^{13} \mathrm{C}$ NMR $\left(126 \mathrm{MHz}\right.$, Acetone- $\left.\mathrm{d}_{6}\right): \delta=143.1,143.1,142.1,142.0,141.6,141.5,141.0$, $140.7,140.2,140.0,136.2,135.9,135.8,130.6,130.5,128.8,128.8,128.7,128.5$, $128.4,127.6,126.8,126.7,126.7,126.5,126.42,125.8,122.9,122.0,120.5,120.4$ $120.4,113.4,112.9,112.7,112.7 . \mathrm{MS}$ (APCI) calcd. for $\mathrm{C}_{91} \mathrm{H}_{57} \mathrm{~N}_{5}: m / z=1220.5$; found: $1221.0[\mathrm{M}]^{+}$. Elemental analysis calcd. (\%) for $\mathrm{C}_{91} \mathrm{H}_{57} \mathrm{~N}_{5}$ : C 89.55, H 4.71, N 5.74; found: C 89.51, H 4.65, N 5.72 .

Synthesis of 4CzBN. Under nitrogen atmosphere, $9 H$-carbazole ( $668 \mathrm{mg}, 4 \mathrm{mmol}$ ) was dissolved in dry $N, N$-dimethylformamide $(30 \mathrm{~mL})$ in a two-neck round-bottom flask equipped with a condenser. The reaction mixture was cooled to $0{ }^{\circ} \mathrm{C}$, then 

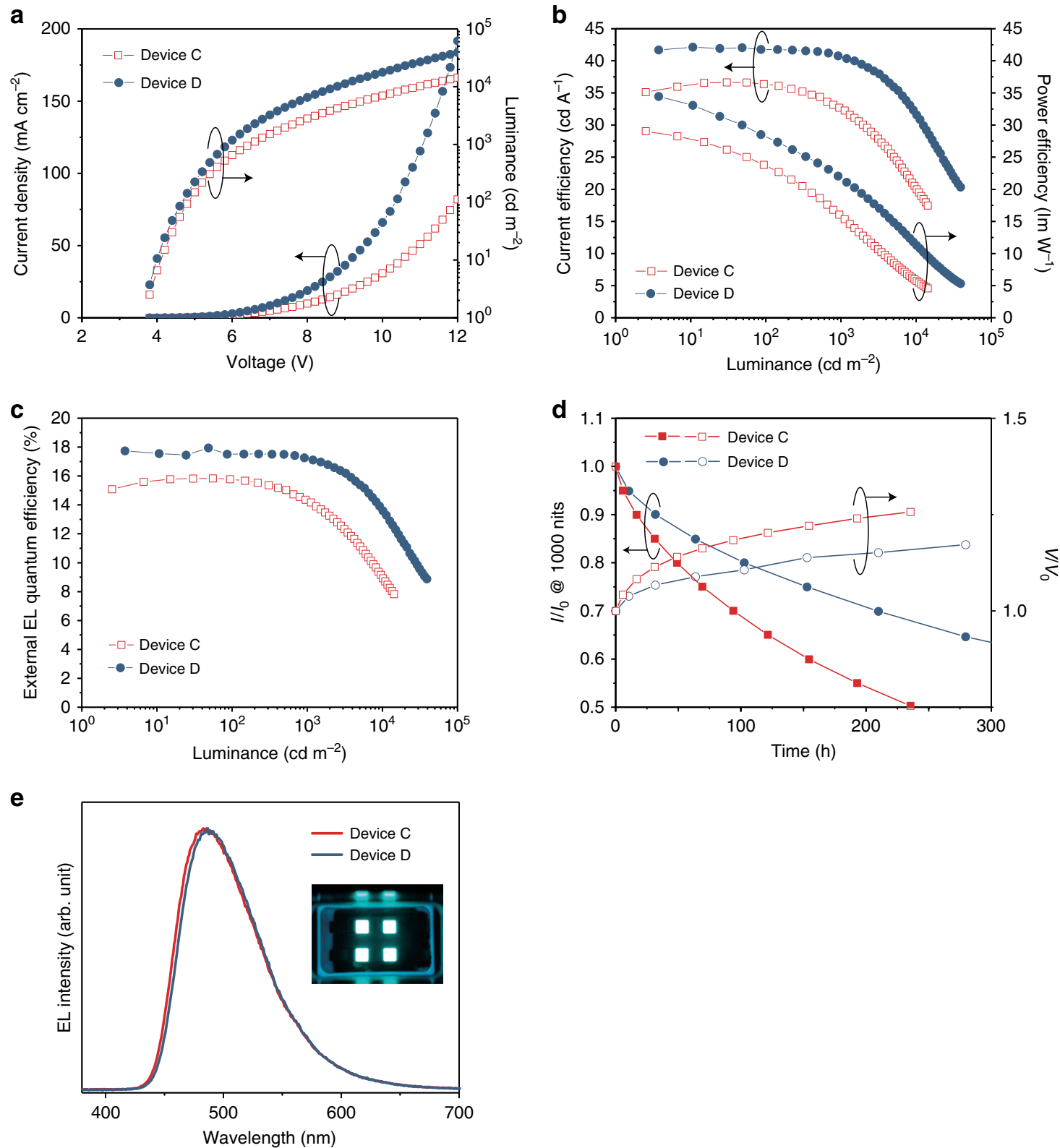

Fig. 3 Device characteristics of device $\mathbf{C}$ and $\mathbf{D}$. a Current density and luminance versus voltage. $\mathbf{b}$ Power efficiency and current efficiency versus luminance. c External quantum efficiency versus luminance. $\mathbf{d}$ Luminance and voltage versus time (at an initial luminance of $1000 \mathrm{~cd} \mathrm{~m}^{-2}$ ). Device structure: ITO (100 nm)/ HATCN (10 nm)/ Tris-PCz (30 nm)/ mCBP ( $5 \mathrm{~nm}) / 15$ wt \% of 3Ph2CzCzBN (device C) or 20 wt\% of 3Ph2CzCzBN (device D): mCBP (30 nm)/ SF3-TRZ $(10 \mathrm{~nm}) / 30$ wt\% of Liq:SF3-TRZ $(50 \mathrm{~nm}) / \mathrm{Liq}(2 \mathrm{~nm}) / \mathrm{Al}(100 \mathrm{~nm})$. e EL spectra of device C and D and photo of the resulting OLED

$\mathrm{NaH}(160 \mathrm{mg}, 4 \mathrm{mmol})$ was added. The reaction mixture was slowly warmed to room temperature and stirred for half an hour. After that, 2,3,5,6-tetrafluorobenzonitrile ( $175 \mathrm{mg}, 1 \mathrm{mmol})$ was added and the reaction was heated to 150 ${ }^{\circ} \mathrm{C}$ for $16 \mathrm{~h}$. The reaction was quenched with water and the precipitate was filtered off. The crude product was purified by column chromatography. Yield: $557 \mathrm{mg}$ (73\%). ${ }^{1} \mathrm{H}$ NMR $\left(500 \mathrm{MHz}, \mathrm{CDCl}_{3}, 298 \mathrm{~K}\right.$, relative to $\left.\mathrm{Me}_{4} \mathrm{Si}\right): \delta=8.43(\mathrm{~s}, 1 \mathrm{H})$, 7.77-7.79(m, $8 \mathrm{H}), 7.35(\mathrm{~d}, 4 \mathrm{H}, 8.0 \mathrm{~Hz}), 7.30-7.32(\mathrm{~m}, 4 \mathrm{H}), 7.18(\mathrm{t}, 4 \mathrm{H}, 7.5 \mathrm{~Hz})$, 7.09-7.17 (m, $12 \mathrm{H}) .{ }^{13} \mathrm{C}$ NMR $\left(126 \mathrm{MHz}, \mathrm{CDCl}_{3}\right): \delta=139.2,138.8,137.8,136.9$, $136.3,125.7,124.3,123.9,121.3,121.0,120.4,120.2,118.3,113.0,109.9,109.2$. MS (APCI) calcd. for $\mathrm{C}_{55} \mathrm{H}_{33} \mathrm{~N}_{5}: \mathrm{m} / z=763.3$; found: $763.3[\mathrm{M}]^{+}$. Elemental analysis calcd. (\%) for $\mathrm{C}_{55} \mathrm{H}_{33} \mathrm{~N}_{5}$ : C 86.48, H 4.35, N 9.17; found: C 86.50, H 4.29, N 9.18.

Quantum chemical calculation. All calculations were carried out using the Gaussian 09 program package. The geometries in the ground state were optimized via TD-DFT calculations at the B3LYP/6-31 G(d) level. TD-DFT calculations for the $S_{0} \rightarrow S_{1}$ and $S_{0} \rightarrow T_{1}$ transitions using the B3LYP functional were then performed according to the optimized geometries of the lowest-lying singlet and triplet states, respectively.
Photophysical measurements. Toluene solutions containing these two materials $\left(10^{-5} \mathrm{M}\right)$ were prepared to investigate their absorption and photoluminescence characteristics in the solution state. Thin-film samples (15\% wt- doped in mCBP $100 \mathrm{~nm}$ ) were deposited on quartz glass substrates by vacuum evaporation to study their exciton confinement properties in the film state. Ultraviolet-visible absorption (UV-vis) and photoluminescence (PL) spectra were recorded on a Perkin-Elmer Lambda 950 KPA spectrophotometer and a JobinYvon FluoroMax-3 fluorospectrometer. Phosphorescent spectra were recorded on a JASCO FP-6500 fluorescence spectrophotometer at $77 \mathrm{~K}$. Absolute PL quantum yields were measured on a Quantaurus-QY measurement system (C11347-11, Hamamatsu Photonics) under nitrogen flow and all samples were excited at 360 $\mathrm{nm}$. The transient PL decay characteristics of solution samples were recorded using a Quantaurus-Tau fluorescence lifetime measurement system (C11367-03, Hamamatsu Photonics). The prompt and delayed PL spectra of the samples were measured under a vacuum using a streak camera system (Hamamatsu Photonics, C4334) equipped with a cryostat (Iwatani, GASESCRT-006-2000, Japan). A nitrogen gas laser (Lasertechnik Berlin, MNL200) with an excitation wavelength of $337 \mathrm{~nm}$ was used. 
Table 2 Device performance of OLEDs based on $3 \mathrm{Ph}_{2} \mathrm{CzCzBN}$ and $4 \mathrm{CzBN}$

\begin{tabular}{|c|c|c|c|c|c|c|c|}
\hline Device & $\lambda_{\mathrm{EL}}(\mathrm{nm})^{\mathrm{a}}$ & $V(V)^{b}$ & EQE $(\%)^{c}$ & PE $\left(\operatorname{Im~} W^{-1}\right)^{d}$ & $C E\left(\operatorname{cd~} A^{-1}\right)^{e}$ & CIE $(x, y)^{f}$ & LT (h) $\mathrm{g}$ \\
\hline A & 482 & $4.0 / 5.0 / 6.3$ & $15.9 / 15.8 / 15.0$ & $27.8 / 22.7 / 17.0$ & $36.3 / 36.0 / 33.9$ & $(0.17,0.37)$ & $4 / 15$ \\
\hline B & 477 & $4.0 / 5.3 / 7.6$ & $9.7 / 8.7 / 5.8$ & $20.9 / 11.3 / 4.9$ & $26.6 / 20.0 / 12.1$ & $(0.19,0.32)$ & $0.1 / 0.4$ \\
\hline C & 482 & $3.7 / 4.8 / 6.4$ & $15.9 / 15.8 / 14.3$ & $29.2 / 23.5 / 15.7$ & $36.6 / 36.2 / 32.6$ & $(0.18,0.37)$ & $16 / 47$ \\
\hline E & 482 & $4.0 / 4.5 / 5.5$ & $17.8 / 17.6 / 16.6$ & $33.9 / 29.1 / 20.7$ & $39.2 / 38.9 / 36.1$ & $(0.17,0.36)$ & $38 / 118$ \\
\hline
\end{tabular}

aEmission peak maximum at $1000 \mathrm{~cd} \mathrm{~m}^{-2}$.

bVoltage at onset, $100 \mathrm{~cd} \mathrm{~m}^{-2}$ and $1000 \mathrm{~cd} \mathrm{~m}^{-2}$

'External Quantum Efficiency: maximum, value at $100 \mathrm{~cd} \mathrm{~m}^{-2}$, value at $1000 \mathrm{~cd} \mathrm{~m}^{-2}$

dPower Efficiency: maximum, value at $100 \mathrm{~cd} \mathrm{~m}^{-2}$, value at $1000 \mathrm{~cd} \mathrm{~m}^{-2}$

eCurrent Efficiency: maximum, value at $100 \mathrm{~cd} \mathrm{~m}^{-2}$, value at $1000 \mathrm{~cd} \mathrm{~m}^{-2}$

fAt $5 \mathrm{~mA} \mathrm{~cm}^{-2}$

g Lifetime (at an initial luminance of $1000 \mathrm{~cd} \mathrm{~m}^{-2}$ ): $\mathrm{LT}_{90} / \mathrm{LT}_{80}$

Thermal properties. Thermal gravimetry-differential thermal analysis (TG-DTA) was performed by Bruker TG-DTA $2400 \mathrm{SA}$ with a heating rate of $10^{\circ} \mathrm{C} \mathrm{min} \mathrm{mi}^{-1}$ under nitrogen atmosphere. Differential scanning calorimetry (DSC) analysis was performed by Netzsch DSC204 Phoenix calorimeter at a scanning rate of $5{ }^{\circ} \mathrm{C} \mathrm{min} \mathrm{min}^{-1}$ under $\mathrm{N}_{2}$ atmosphere.

Cyclic voltammetry measurements. Cyclic voltammetry $(\mathrm{CV})$ was carried out on a CHI600 voltammetric analyzer at room temperature with a conventional threeelectrode configuration consisting of a platinum disk working electrode, a platinum wire auxiliary electrode and an Ag wire pseudo-reference electrode with ferrocenium-ferrocene $\left(\mathrm{Fc}^{+} / \mathrm{Fc}\right)$ as the internal standard. Argon-purged $N, N$ dimethylformamide was used as solvent for scanning the oxidation with tetrabutylammonium hexafluorophosphate $\left(\mathrm{TBAPF}_{6}\right)(0.1 \mathrm{M})$ as the supporting electrolyte. The cyclic voltammograms were obtained at a scan rate of $100 \mathrm{mV} \mathrm{s}^{-1}$.

Device fabrication and measurements. The OLEDs were fabricated through vacuum deposition of the materials at ca. $2.0 \times 10^{-4} \mathrm{~Pa}$ onto indium-tin-oxidecoated glass substrates having a sheet resistance of ca. $15 \Omega$ per square. The indium-tin oxide surface was cleaned ultrasonically and sequentially with acetone, isopropanol and deionized water, then dried in an oven, and finally exposed to ultraviolet light and ozone for about $10 \mathrm{~min}$. Organic layers and aluminum were deposited at a rate of 1-2 $\mathrm{A} / \mathrm{s}$. Subsequently, LiF and Liq were deposited at $0.1-0.2$ $\AA /$ s. The devices were exposed once to nitrogen gas after the formation of the organic layers to allow the fixing of a metal mask to define the cathode area. After fabrication, the devices were immediately encapsulated with glass lids using epoxy glue in nitrogen-filled glove boxes $\left(\mathrm{O}_{2}-0.1 \mathrm{ppm}, \mathrm{H}_{2} \mathrm{O}-0.1 \mathrm{ppm}\right)$. For all OLEDs, the emitting areas were determined by the overlap of two electrodes as $0.04 \mathrm{~cm}^{2}$. The JV-luminance characteristics were evaluated using a Keithley 2400 source meter and an absolute external quantum efficiency (EQE) measurement system (C9920-12, Hamamatsu Photonics, Japan). Device operational stability was measured using a luminance meter (CS-2000, Konica Minolta, Japan) at a constant DC current at room temperature.

Optical simulation. The optical simulation of OLED devices was performed by using SETFOS 4.6 simulation program, in which the corresponding refractive index (n) and extinction coefficient (k) of each layer, dipole orientation factor of emitter, thickness of each layer and emission spectrum of EML were input. The optical constants of all the organic layers were measured using ellipsometer (M-2000, J. A Woollam). The dipole orientation factor of emitter was set to be isotropic. During the optical simulation, the thicknesses of all layers were fixed, except the thickness of ETL was varied from 10 to $60 \mathrm{~nm}$.

\section{Data availability}

The data that support the plots within the paper are available from the corresponding author upon reasonable request.

Received: 26 June 2018 Accepted: 5 November 2018

Published online: 28 November 2018

\section{References}

1. Tang, C. W. \& VanSlyke, S. A. Organic electroluminescent diodes. Appl. Phys. Lett. 51, 913-915 (1987).

2. Rogers, J. A., Someya, T. \& Huang, Y. Materials and mechanics for stretchable electronics. Science 327, 1603-1607 (2010).
3. White, M. et al. Ultrathin, highly flexible and stretchable PLEDs. Nat. Photon. 7, 811-816 (2013)

4. Kaltenbrunner, M. et al. An ultra-lightweight design for imperceptible plastic electronics. Nature 499, 458-463 (2013).

5. Sekitani, T. et al. Stretchable active-matrix organic light-emitting diode display using printable elastic conductors. Nat. Mater. 8, 494-499 (2009).

6. Yook, K. S. \& Lee, J. Y. Organic materials for deep blue phosphorescent organic light-emitting diodes. Adv. Mater. 24, 3169-3190 (2012).

7. Im, Y. et al. Recent progress in high-efficiency blue-light-emitting materials for organic light-emitting diodes. Adv. Funct. Mater. 27, 1603007 (2017).

8. Zhu, M. \& Yang, C. Blue fluorescent emitters: design tactic and applications in organic light-emitting diodes. Chem. Soc. Rev. 42, 4963-4976 (2013).

9. Yang, X., Xu, X. \& Zhou, G. Recent advances of the emitters for high performance deep-blue organic light-emitting diodes. J. Mater. Chem. C. 3, 913-944 (2015).

10. Chan, C.-Y., Cui, L.-S., Kim, J. K., Nakanotani, H. \& Adachi, C. Rational molecular design for deep-blue thermally activated delayed fluorescence emitters. Adv. Funct. Mater. 28, 1706023 (2018).

11. Nakanotani, H. et al. High-efficiency organic light-emitting diodes with fluorescent emitters. Nat. Commun. 5, 4016 (2014).

12. Wu, T.-L. et al. Diboron compound-based organic light-emitting diodes with high efficiency and reduced efficiency roll-off. Nat. Photon. 12, 235-240 (2018).

13. Kuei, C. Y. et al. Bis tridentate Ir(III) complexes with nearly unitary RGB efficiency exceeding 31\%. Adv. Mater. 28, 2795-2800 (2016).

14. Kim, K. H., Ahn, E. S., Huh, J. S., Kim, Y. H. \& Kim, J. J. Design of heteroleptic Ir complexes with horizontal emitting dipoles for highly efficient organic light-emitting diodes with an external quantum efficiency of $38 \%$. Chem. Mater. 28, 7505-7510 (2016).

15. Lin, T.-A. et al. Sky-blue organic light emitting diode with $37 \%$ external quantum efficiency using thermally activated delayed fluorescence from spiroacridine-triazine hybrid. Adv. Mater. 28, 6976-6983 (2016).

16. Udagawa, K., Sasabe, H., Cai, C. \& Kido, J. Low-driving-voltage blue phosphorescent organic light-emitting devices with external quantum efficiency of 30\%. Adv. Mater. 26, 5062-5066 (2014).

17. Sasabe, H. et al. 3,3'-Bicarbazole-based host materials for high-efficiency blue phosphorescent OLEDs with extremely low driving voltage. Adv. Mater. 24, 3212-3217 (2012)

18. Udagawa, K., Sasabe, H., Igarashi, F. \& Kido, J. Simultaneous realization of high $\mathrm{EQE}$ of $30 \%$, low driving voltage, and low efficiency roll-off at high brightness in blue phosphorescent OLEDs. Adv. Opt. Mater. 4, 86-90 (2016).

19. Zhang, D. et al. Highly efficient blue thermally activated delayed fluorescent OLEDs with record-low driving voltages utilizing high triplet energy hosts with small singlet-triplet splittings. Chem. Sci. 7, 3355-3363 (2016).

20. Zhang, Y., Lee, J. \& Forrest, S. Tenfold increase in the lifetime of blue phosphorescent organic light-emitting diodes. Nat. Commun. 5, 5008 (2014).

21. Lee, J. et al. Hot excited state management for long-lived blue phosphorescent organic light-emitting diodes. Nat. Commun. 8, 15566 (2017).

22. Sarma, M. et al. Anomalously long-lasting blue PhOLED featuring phenylpyrimidine cyclometalated iridium emitter. Chem 3, 461-476 (2017).

23. Zhang, D., Cai, M., Zhang, Y., Zhang, D. \& Duan, L. Sterically shielded blue thermally activated delayed fluorescence emitters with improved efficiency and stability. Mater. Horiz. 3, 145-151 (2016).

24. Cui, L.-S. et al. Long-lived efficient delayed fluorescence organic light-emitting diodes using n-type hosts. Nat. Commun. 8, 2250 (2017).

25. Kim, M., Jeon, S. K., Hwang, S.-H. \& Lee, J. Y. Stable blue thermally activated delayed fluorescent organic light-emitting diodes with three times longer lifetime than phosphorescent organic light-emitting diodes. Adv. Mater. 27, 2515-2520 (2015). 
26. Nakanotani, H., Masui, K., Nishide, J., Shibata, T. \& Adachi, C. Promising operational stability of high-efficiency organic light-emitting diodes based on thermally activated delayed fluorescence. Sci. Rep. 3, 2127 (2013).

27. Tsang, D. P.-K., Matsushima, T. \& Adachi, C. Operational stability enhancement in organic light-emitting diodes with ultrathin Liq layers. Sci. Rep. 6, 22463 (2016)

28. Adachi, C. et al. Endothermic energy transfer: a mechanism for generating very efficient high-energy phosphorescent emission in organic materials. Appl. Phys. Lett. 79, 2082-2084 (2001).

29. Klubek, K. P., Dong, S.-C., Liao, L.-S., Tang, C. W. \& Rothberg, L. J. Investigating blue phosphorescent iridium cyclometalated dopant with phenyl-imidazole ligands. Org. Electron. 15, 3127-3136 (2014).

30. Zhuang, J. et al. Highly efficient phosphorescent organic light-emitting diodes using a homoleptic iridium(III) complex as a sky-blue dopant. Org. Electron. 14, 2596-2601 (2013).

31. Uoyama, H., Goushi, K., Shizu, K., Nomura, H. \& Adachi, C. Highly efficient organic light-emitting diodes from delayed fluorescence. Nature 492, 234-238 (2012).

32. Wong, M. Y. \& Zysman-Colman, E. Purely organic thermally activated delayed fluorescence materials for organic light-emitting diodes. Adv. Mater. 29, 160544 (2017)

33. Yang, Z. et al. Recent advances in organic thermally activated delayed fluorescence materials. Chem. Soc. Rev. 46, 915-1016 (2017).

34. Im, Y. et al. Molecular design strategy of organic thermally activated delayed fluorescence emitters. Chem. Mater. 29, 1946-1963 (2017).

35. Giebink, N., DAndrade, B., Weaver, M., Brown, J. \& Forrest, S. Direct evidence for degradation of polaron excited states in organic light emitting diodes. J. Appl. Phys. 105, 124514 (2009).

36. Giebink, N. et al. Intrinsic luminance loss in phosphorescent small-molecule organic light emitting devices due to bimolecular annihilation reactions. J. Appl. Phys. 103, 044509 (2008).

37. Wang, Q. \& Aziz, H. Degradation of organic/organic interfaces in organic light-emitting devices due to polaron-exciton interactions. ACS Appl. Mater. Interfaces 5, 8733-8739 (2013).

38. Noda, H., Nakanotani, H. \& Adachi, C. Excited state engineering for efficient reverse intersystem crossing. Sci. Adv. 4, eaao6910 (2018).

\section{Acknowledgements}

This work was supported financially by the Program for Building Regional Innovation Ecosystems of the Ministry of Education, Culture, Sports, Science and Technology, Japan and JST ERATO Grant Number JPMJER1305, Japan and Kyulux Inc. The computation was mainly carried out using the computer facilities at Research Institute for Information
Technology, Kyushu University. The authors also acknowledge Ms. Nozomi Nakamura and Ms. Keiko Kusuhara for their technical assistance with this research.

\section{Author contributions}

C.A. initiated and supervised the project. C.-Y.C. designed, synthesized and characterized the blue TADF emitters. C.-Y.C. performed the computational calculation, photophysical and electrochemical measurements of the TADF emitters. C.-Y.C. and M.T. fabricated the OLEDs and measured the device performance and stability. C.-Y.C. and C.A. contributed to the manuscript writing. M.T., H.N. and C.A. provided suggestions on experiments and writing manuscript. All authors discussed the progress of the research and reviewed the manuscript.

\section{Additional information}

Supplementary Information accompanies this paper at https://doi.org/10.1038/s41467018-07482-6.

Competing interests: C.A. is the external advisor of one of the sponsors of this work (Kyulux). The remaining authors declare no competing interests.

Reprints and permission information is available online at http://npg.nature.com/ reprintsandpermissions/

Publisher's note: Springer Nature remains neutral with regard to jurisdictional claims in published maps and institutional affiliations.

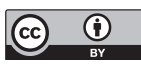

Open Access This article is licensed under a Creative Commons Attribution 4.0 International License, which permits use, sharing, adaptation, distribution and reproduction in any medium or format, as long as you give appropriate credit to the original author(s) and the source, provide a link to the Creative Commons license, and indicate if changes were made. The images or other third party material in this article are included in the article's Creative Commons license, unless indicated otherwise in a credit line to the material. If material is not included in the article's Creative Commons license and your intended use is not permitted by statutory regulation or exceeds the permitted use, you will need to obtain permission directly from the copyright holder. To view a copy of this license, visit http://creativecommons.org/ licenses/by/4.0/.

(c) The Author(s) 2018 